\title{
Propuesta de sinonimia para las especies de cangrejo dulceacuícola Hypolobocera solimani e Hypolobocera triangula de Colombia
}

\author{
Martha R. Campos ${ }^{1} \&$ Luis A. Guerra ${ }^{2}$ \\ 1. Instituto de Ciencias Naturales,Universidad Nacional de Colombia, Bogotá, Colombia; mhrochad@unal.edu.co \\ 2. Sede Caribe, Universidad Nacional de Colombia, San Andrés Isla, Colombia; laguerrava@unal.edu.co
}

Recibido 28-XI-2007. Corregido 30-VI-2008. Aceptado 31-VII-2008.

\begin{abstract}
A proposal to synonymize the freshwater crab species Hypolobocera solimani and Hypolobocera triangula from Colombia. It is proposed that Hypolobocera solimani and $H$. triangula be considered junior synonyms of $H$. alata and $H$. rotundilobata, respectively. We state that the following morphological differences represent natural variations within the population: a) first male gonopod, third maxilliped and larger cheliped between $H$. alata and $H$. solimani, and b) the differences of the first male gonopod between $H$. rotundilobata and H. triangula. Rev. Biol. Trop. 56 (3): 987-994. Epub 2008 September 30.
\end{abstract}

Key words: Hypolobocera solimani, Hypolobocera triangula, freshwater crabs, Pseudothelphusidae, Colombia, junior synonym.

Los caracteres morfológicos del primer par de gonopodos del macho son la base para la determinación taxonómica de las especies de cangrejos de agua dulce (Smalley 1964, Rodríguez 1981). Esta especificidad se debe a que el gonopodo y la abertura femenina funcionan como un sistema llave/cerradura cuya función es la transferencia del esperma a la hembra (Pedraza y Campos 2007).

Elementos esenciales que se deben tener en cuenta en la determinación taxonómica de las especies son los siguientes: (i) Cada género se caracteriza por un patrón general del primer gonopodo del macho (presencia y forma del lóbulo lateral, lóbulo accesorio, lóbulo mesial, procesos, proyecciones). (ii) Una especie, además de compartir el patrón general del género al que pertenece, se distingue por un conjunto de caracteres morfológicos diagnósticos específicos y significativos que le dan identidad y la diferencian claramente de cualquier otra especie del género. (iii) En general, para una especie dada existen diferentes poblaciones influenciadas por las condiciones ambientales, lo que genera pequeñas variaciones en los caracteres morfológicos diagnósticos, sin que por ello la especie pierda su identidad.

Para ilustrar lo anterior, se citan a continuación algunos ejemplos descritos en la literatura: (A) En la revisión del género Neostrengeria Pretzmann, 1965 se ilustran, para algunas especies, variaciones morfológicas del primer gonopodo del macho, esencialmente del lóbulo lateral, del lóbulo accesorio, del ápice y de la proyección meso-caudal del canal espermático, definidas por un patrón poblacional (Campos 1994, Fig. 10 a-f, Fig. 11 a-d, Fig. 15 a-f, Fig. 19 a-c, 25 a-d, Fig. 31 a-f, Fig. 36 a-c). (B) En el género Hypolobocera Ortmann 1897, las variaciones se centran en el lóbulo lateral y en el contorno del ápice (Campos 2003, Fig. 5 A, B, Fig. 17 E, F). (C) Rodríguez (1982) estableció para Microthelphusa recenisi (Rodríguez 1966) cuatro patrones de variación del proceso mesial y del canal espermático, los cuales están directamente correlacionados con el tamaño de los especímenes. $(D)$ Un caso muy particular se presenta en Strengeriana maniformis Campos y 
Rodríguez, 1993, donde en machos adultos se presentan variaciones en el proceso mesial del gonopodo, de tal forma que cada espécimen presenta una forma particular de este proceso (Pedraza y Campos 2007).

Como se ha enfatizado anteriormente, las características del gonopodo son lo fundamental en la determinación taxonómica de las especies, pero existen caracteres complementarios (del caparazón, del tercer maxilípedo, de los pereopodos) que facilitan el proceso de determinación, aunque también presentan variaciones en las diversas poblaciones.

En reciente publicación, Ramos-Tafur (2006) describe dos nuevas especies de cangrejos de agua dulce, pertenecientes al género Hypolobocera: H. solimani Ramos-Tafur, 2006 e H. triangula Ramos-Tafur, 2006, especies simpátricas del occidente de Colombia. El autor fundamenta la determinación taxonómica de estas especies en la morfología del primer gonopodo del macho y establece que las nuevas especies están muy relacionadas con $H$. alata Campos, 1989 e H. rotundilobata Rodríguez, 1994, respectivamente.

En el presente trabajo, al comparar las descripciones e ilustraciones de: $H$. solimani e $H$. triangula, con material de $H$. alata e $H$. rotundilobata de las colecciones de referencia de Crustáceos del Instituto de Ciencias Naturales, del National Museum of Natural History, Smithsonian Institution, Washington (USNM), de Biología Marina, Universidad del Valle (CRBMUV), se concluye que las diferencias morfológicas del primer gonopodo del macho corresponden sólo a variaciones poblacionales de las especies $H$. alata e $H$. rotundilobata,. Es decir, Ramos-Tafur (2006) no tuvo en cuenta el elemento esencial (iii) antes mencionado. En consecuencia, $H$. solimani e $H$. triangula se constituyen en sinónimos de las especies $H$. alata e $H$. rotundilobata, respectivamente. Se procede ahora a fundamentar esta afirmación.

La terminología que se emplea para el primer gonopodo del macho se basa en Smalley (1964) y Campos (2003, 2005). Las medidas que se registran como cl, cb, corresponden a longitud y ancho del caparazón. El material examinado se encuentra en la colecciones de referencia, del Instituto de Ciencias Naturales, Universidad Nacional de Colombia, Bogotá (ICN-MHN), del National Museum of Natural History, Smithsonian Institution, Washington (USNM) y de Biología Marina, Universidad del Valle (CRBMUV).

\section{Hypolobocera alata Campos, 1989}

Fig. $1 \mathrm{~A}-\mathrm{E}$

Hypolobocera alata Campos, 1989: 145, Fig. 2 a-g.

Hypolobocera alata, Rodríguez, 1992:183.- Campos, 2003: 755, 756, 797800, Fig. 1 A-F. - Campos, 2005: 227, Fig. $67 \mathrm{~A}-\mathrm{H}$.

Hypolobocera solimani Ramos-Tafur, 2006: 1048, Fig. 1 A-F, Fig. 2 A-C.

Material examinado: Colombia. Chocó, Tadó, Corregimiento Guarató, quebrada Guadralito, 500 m alt., 28 Sep 1991, leg. G. Andrade, 1 macho, cl $12.1 \mathrm{~mm}$, cb $19.4 \mathrm{~mm}$, ICN-MHN-CR 1308. - Huila, Villavieja, quebrada La Batea, 400 m alt., 3 Abr 1982, leg. R. Restrepo, 1 macho holotipo, cl $13.1 \mathrm{~mm}$, cb $20.2 \mathrm{~mm}, 1$ hembra paratipo, cl $13.4 \mathrm{~mm}$, cb $21.4 \mathrm{~mm}$, ICN-MHN-CR 0853.- - Risaralda, Pueblo Rico, Corregimiento Santa Cecilia, quebrada Amurropa, 490 m alt., 26 Sep 1991, leg. G. Andrade, 2 machos, cl 12.3 y $10.1 \mathrm{~mm}$, cb 19.5 y 15.2 mm, ICN-MHN-CR 1309.Pueblo Rico, Corregimiento Santa Cecilia, Vereda La Granja, $700 \mathrm{~m}$ alt., 22 Oct 1991, leg. M. R. Campos, 1 macho, cl $12.1 \mathrm{~mm}$, cb 19.6 mm, ICN-MHN-CR 1306.

Diagnosis: Primer gonopodo angosto, curvado en dirección caudocefálica; porción distal del márgen caudal con hilera de espinas romas; cresta caudal protuberante, casi recta, curvada distalmente, termina más allá del lóbulo lateral; lóbulo lateral triangular; contorno del ápice ligeramente ovalado en vista apical, inclinado fuertemente en dirección caudo-cefálica, borde caudo-lateral proyectado distalmente, redondeado con 4 o 5 espinas semi-agudas, 

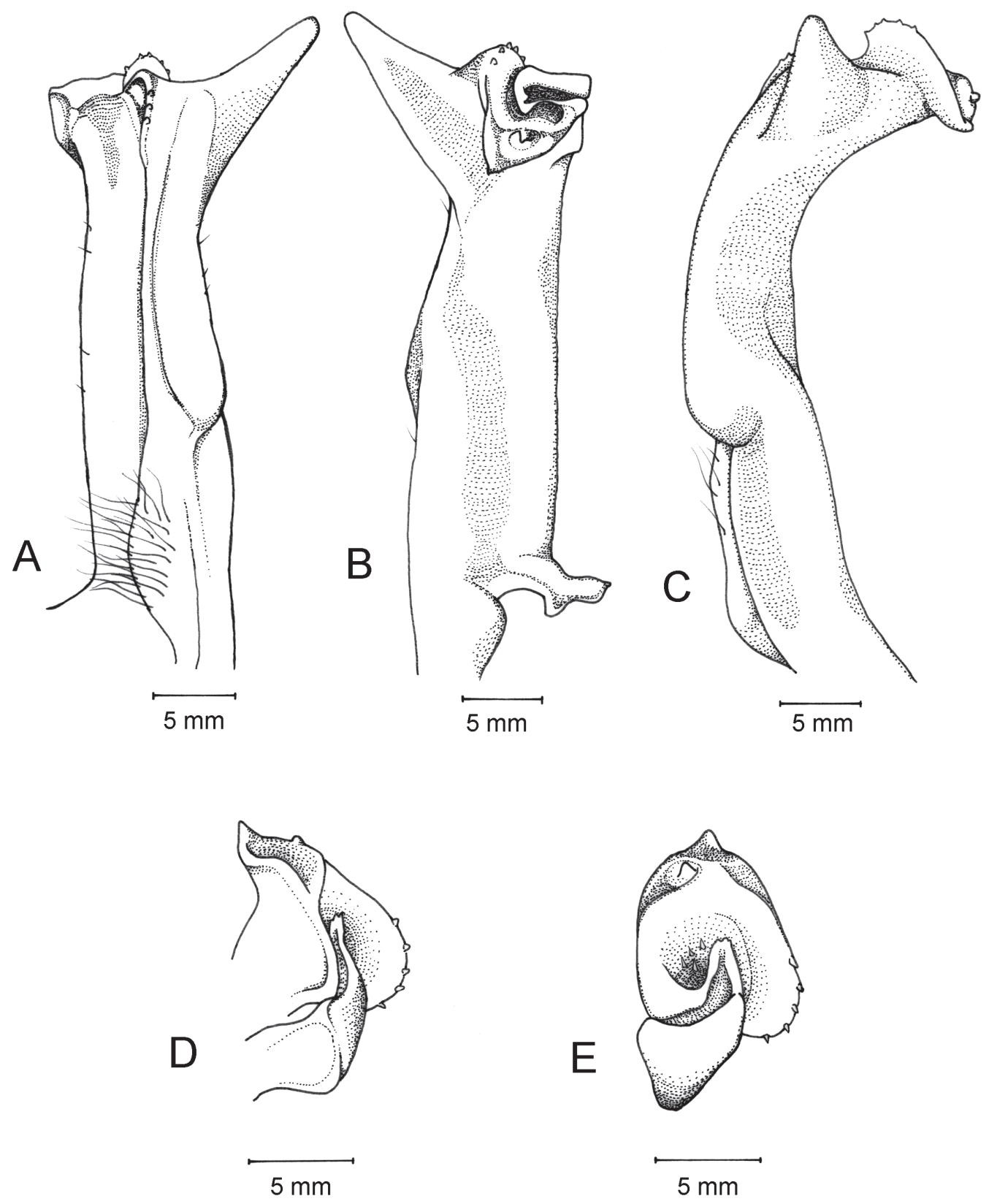

Fig. 1. Hypolobocera alata Campos, 1989, macho holotipo, ICN-MHN-CR 0853. A. gonopodo izquierdo, vista caudal. B. Mismo, vista cefálica. C. Mismo, vista lateral. D. Detalle de la porción apical del gonopodo, vista mesial. E. Apice del gonopodo, vista distal.

Fig. 1. Hypolobocera alata Campos, 1989, male holotype, ICN-MHN-CR 0853. A. Left gonopod, caudal view. B. Same, cephalic view. C. Same, lateral view. D. Detail of apical portion of gonopod, mesial view. E. Apex of gonopod, distal view. 
borde latero-cefálico expandido en forma de una proyección subtriangular, doblada hacia abajo, con dos papilas: una papila redondeada terminal y una papila pequeña en la parte superior; la proyección mesocaudal del canal espermático cóncava, terminada en una papila ligeramente bífida y 1 papila anexa o de forma redondeada; lóbulo mesial subtriangular con su porción distal curvada caudalmente. Tercer maxilipedo con exognato 0.25 a 0.35 veces la longitud del isquio del endognato.

Comentarios: Al examinar el material de Hypolobocera alata de la colección del Instituto de Ciencias Naturales y compararlo con la descripción e ilustraciones del holotipo de H. solimani, se observa que la mayoría de las características morfológicas de ambas especies coinciden. En lo referente al primer gonopodo del macho, se tienen las siguientes similitudes y diferencias, aunque es de anotar que debido a la inclinación y torsión del ápice respecto al eje longitudinal del gonopodo en $H$. alata, los bordes en el ápice no corresponden exactamente a cada una de los planos de las vistas. Por tanto hay que considerar los bordes caudo-lateral, latero-cefálico, cefalo-mesial y meso-caudal. El borde caudo-lateral del ápice en $H$. alata es redondeado y se hace casi recto hacia el borde latero-cefálico (Fig. 1E), similar a H. solimani (Ramos-Tafur 2006, Fig.1F). A excepción de un macho del lote (ICN-MHNCR 1309) de $H$. alata, que sólo presenta 4 espinas semi-agudas, en el borde caudo-lateral (Campos 1993, Fig. 1D), en el resto de especímenes examinados de $H$. alata el número de espinas es de 5 (Fig. 1E), lo cual coincide con H. solimani (Ramos-Tafur 2006, Fig.1F). Tanto en H alata como en H. solimani el borde laterocefálico es expandido en forma de una proyección subtriangular, doblada hacia abajo, con dos papilas: una papila redondeada terminal, proyectada lateralmente, y una papila pequeña en la parte superior (Fig. 1D, E); la diferencia radica en que en $H$. solimani la papila terminal se encuentra curvada hacia arriba (RamosTafur 2006, Fig. 1E), mientras que en $H$. alata está proyectada lateralmente (Fig. 1D). Es importante aclarar que Ramos-Tafur (2006) considera esta papila terminal como un diente. La proyección meso-caudal del canal espermático en $H$. alata es cóncava con su parte distal ligeramente hacia arriba, terminada en una papila ligeramente bífida (Campos 1993, Fig. 1D) y con una papila anexa (Fig. 1D, E) o de forma redondeada (ICN-MHN-CR 1309), mientras que en $H$. solimani la porción distal está curvada hacia arriba y el borde interno bordeado por diminutas papilas (Ramos-Tafur 2006, Fig. 1 E, F).

En lo que se refiere a los caracteres complementarios la comparación indica que la mayoría de las características morfológicas de ambas especies coinciden. Sólo se presentan las siguientes diferencias: $H$. alata tiene el borde superior de la frente con algunos pequeños tubérculos, observables en vista dorsal y frontal, mientras que en $H$. solimani es liso. El tercer maxilipedo en $H$. alata presenta una depresión profunda en la porción subdistal externa del márgen del mero (ICN-MHNCR 0853, 1308, 1309), pero esta es somera en un macho del lote (ICN-MHN-CR 1309), lo cual se aproxima a lo ilustrado para $H$. solimani, cuyo margen externo no presenta esta depresión (Ramos-Tafur 2006, Fig. 1 C); asi mismo, la relación del exognato de 0.25 veces la longitud del isquio del endognato en $H$. solimani, se encuentra en el ámbito de 0.25 a 0.35 , establecido para los especímenes de H. alata examinados. El dactilo y el dedo fijo de la quela mayor son de igual longitud, tanto en los machos como en las hembras de H. alata, mientras que en el holotipo y alotipo de H. solimani el dactilo es más corto que el dedo fijo.

Las variaciones morfológicas descritas anteriormente para el primer gonopodo del macho corresponden a variaciones poblacionales propias de la especie, que no alteran el patrón general de $H$. alata. Así mismo, los caracteres complementarios (del caparazón, del tercer maxilípedo y de los pereopodos) no se modifican sustancialmente con respecto a los caracteres de H. alata. En conclusión, 
Hypolobocera solimani se debe considerar como sinónimo de $H$. alata.

Campos (1989) registró como la localidad típica de la especie Hypolobocera alata, Departamento del Huila, Municipio de Villavieja, Quebrada La Batea, 400 m de elevación en el valle del Magdalena. Posteriormente, Campos (2003) señala que esta localidad puede ser errónea y establece con base en nuevos registros, que la distribución de esta especie es entre Pueblo Rico, Departamento de Risaralda, alto río San Juan y Tadó, Departamento del Chocó, medio río San Juan. El nuevo registro amplía la distribución de la especie hasta el Municipio de El Cairo en el Departamento del Valle del Cauca. Así mismo, el ámbito altitudinal inicialmente de 490 a $700 \mathrm{~m}$, se amplia de $490 \mathrm{~m}$ a $2500 \mathrm{~m}$.

\section{Hypolobocera rotundilobata Rodríguez, 1994}

Fig. 2 A - E

Hypolobocera bouvieri rotundilobata Rodríguez, 1994: 297, Fig. 2 A-C.

Hypolobocera bouvieri rotundilobata, Rodríguez et al., 2002: 6.

Hypolobocera rotundilobata, Campos, 2003: 791, 794, 795, Fig. 22 A-H.

Hypolobocera triangula Ramos-Tafur, 2006: 1051, Fig. 3 A-E, Fig. 4 A-C.

Material: Colombia. Chocó, Montañas del Alto río San Juan, cerca de Playa de Oro, 28 Mar 1962, leg. M. Latham, 1 macho holotipo, cl $46.2 \mathrm{~mm}$, cb $68.4 \mathrm{~mm}$, USNM 240103. - Localidad igual a la del holotipo: 5 machos paratipos, el mayor cl $15.4 \mathrm{~mm}$, cb $26.3 \mathrm{~mm}$, el menor cl $12.1 \mathrm{~mm}$, cb $22.6 \mathrm{~mm}$, 1 hembra, cl $18.0 \mathrm{~mm}$, cb $31.6 \mathrm{~mm}$, USNM 240104.- Chocó, San José del Palmar, río Ingará, $2000 \mathrm{~m}$ alt., 22 Oct 2000, leg. E. Guerra, 1 macho, cl $13.1 \mathrm{~mm}, \mathrm{cb} 22.8 \mathrm{~mm}, 1$ hembra, cl $13.3 \mathrm{~mm}$, cb $22.9 \mathrm{~mm}$, ICN-MHNCR 1867, 1868. Límites entre Chocó y Valle del Cauca, Paso de Galápagos, 15 May 1985, leg. M. Alberico, 2 machos, cl 13.3 y 12.4 $\mathrm{mm}$, cb 23.3 y $21.1 \mathrm{~mm}$ y 6 hembras, la mayor cl $13.1 \mathrm{~mm}$, cb $22.6 \mathrm{~mm}$, la menor cl $10.2 \mathrm{~mm}$, cb 17.2 mm, CRBMUV 85147.

Diagnosis: Quela mayor del macho desprovista de tubérculo en la base externa de los dedos fijo y móvil. Primer gonopodo con la cresta caudal larga, recta, angosta distalmente; margen caudal con expansión redondeada en la porción subdistal; lóbulo lateral redondeado o subtriangular; cresta de tubérculos en el margen cefálico presente o ausente; superficie cefálica con cresta transversal bordeada o no de tubérculos; contorno del ápice subtriangular en vista distal, borde lateral recto o ligeramente redondeado, borde cefálico liso o con una hilera de pequeñas espinas y una papila redondeada o semi-aguda; projección meso-caudal del canal espermático terminada en papila redondeada, semiaguda o ligeramente bífida; lóbulo mesial subtriangular y proyectado mesialmente. Tercer maxilipedo con exognato 0.3 a 0.4 veces la longitud del isquio del endognato.

Comentarios: Se examinó material de Hypolobocera rotundilobata de las colecciones de referencia del Instituto de Ciencias Naturales, de Biología Marina, Universidad del Valle (CRBMUV), uno de los paratipos, USNM 240104, del National Museum of Natural History, Smithsonian Institution, Washington (USNM) (Campos 2003, Fig. 22 A-H) y se revisó la descripción e ilustraciones del holotipo, USNM 240103 (Rodríguez 1994, Fig. 2 A-C). Es importante aclarar que las ilustraciones de $H$. rotundilobata (Fig. 22, Campos 2003) realmente corresponden a uno de los paratipos y no al holotipo, como se menciona en la leyenda de la figura.

En lo que se refiere a los caracteres complementarios (del caparazón, del tercer maxilípedo y de los pereopodos) la comparación indica que las características morfológicas de ambas especies coinciden.

Con respecto al primer gonopodo del macho se determinaron las siguientes similitudes y diferencias entre ambas especies. El lóbulo lateral del primer gonopodo es redondeado en el holotipo de $H$. rotundilobata, 

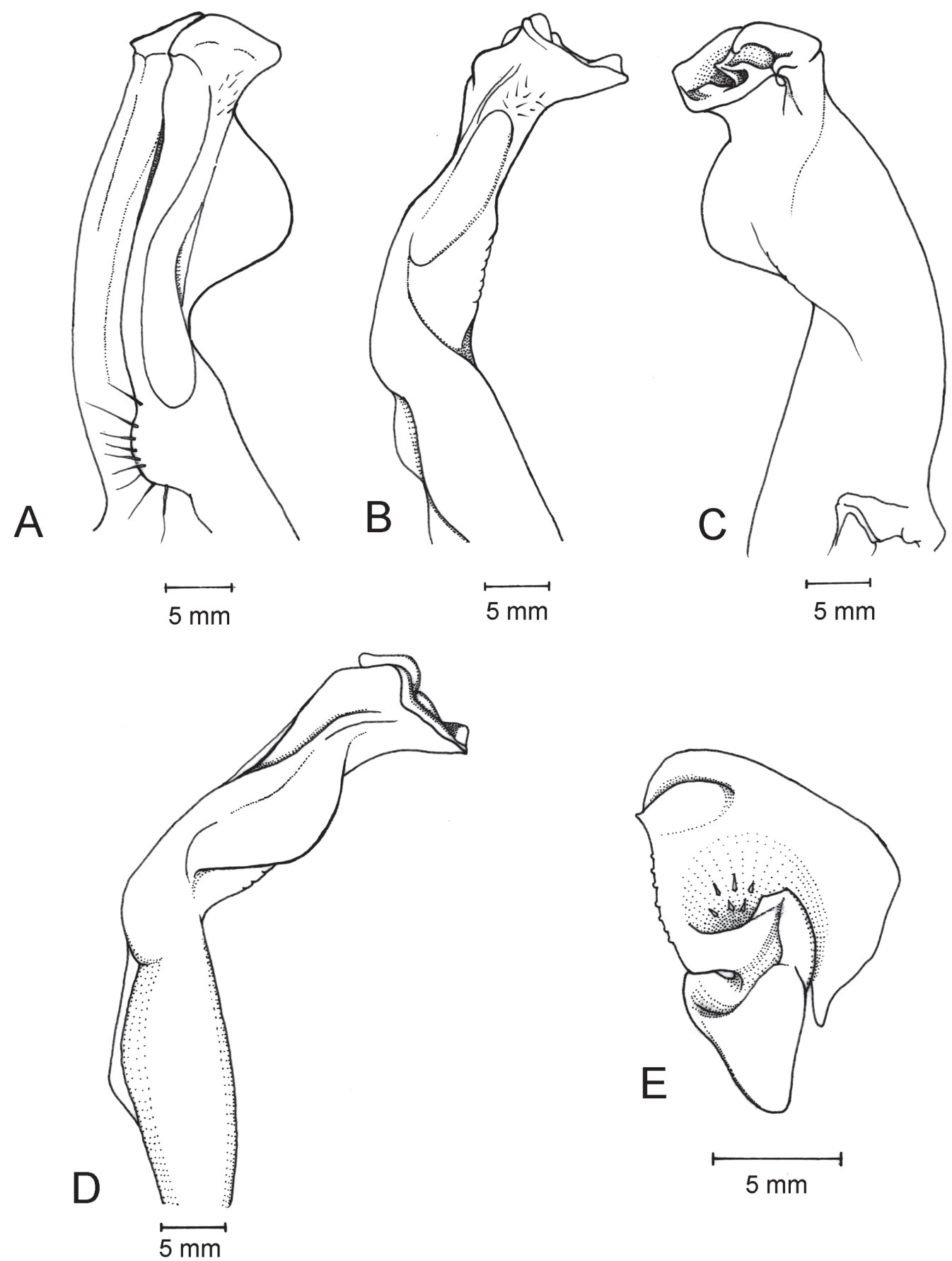

Fig. 2. Hypolobocera rotundilobata Rodríguez, 1994, macho, ICN-MHN-CR 1867. A. gonopodo izquierdo, vista caudal. B. Mismo, vista lateral. C. Mismo, vista cefálica. D. Mismo, vista caudo-lateral. E. Apice del gonopodo, vista distal.

Fig. 2. Hypolobocera rotundilobata Rodríguez, 1994, male, ICN-MHN-CR 1867. A. Left gonopod, caudal view. B. Same, lateral view. C. Same, cephalic view. D. Same, caudo-lateral view. E. Apex of gonopod, distal view. 
(Rodríguez 1994, Fig. 2 A-C), mientras que es subtriangular en el paratipo de $H$. rotundilobata (Campos 2003, Fig. 22 A), en el holotipo de $H$. triangula (Ramos-Tafur 2006, Fig. 4 C) y en el espécimen ilustrado en el presente trabajo (ICN-MHN-CR 1867, Fig. 2 E), de tal manera que el lóbulo lateral en $H$. rotundilobata puede variar desde redondeado a subtriangular. La proyección en forma de joroba de la cresta caudal del gonopodo que Ramos-Tafur (2006, Fig. 4 C) describe para $H$. triangula, realmente corresponde a una expansión de forma redondeada de la porción subdistal del margen (Fig. 2 B, D), la cual se observa también en el paratipo (Campos 2003, Fig. 22 B, D). Sobre la superficie cefálica del gonopodo del los holotipos de H. rotundilobata (Rodríguez 1994, Fig. 2 C), de H. triangula (Ramos-Tafur 2006, Fig. 4 B) y del espécimen ilustrado en el presente trabajo (ICN-MHN-CR 1867, Fig. 2 B-E) se observa una cresta transversal bordeada de tubérculos que se extiende desde la parte media del lóbulo lateral hasta cerca de la mitad del gonopodo. Esta cresta se observa también en el paratipo de $H$. rotundilobata, pero difiere de los anteriores en que carece del reborde de tubérculos (Campos 2003, Fig. 22 B -D). El margen cefálico del holotipo de $H$. rotundilobata presenta una cresta con tubérculos (Rodríguez 1994, Fig. 2 B), mientras que esta cresta está ausente en el paratipo (Campos 2003, Fig. 22 B), en H. triangula (Ramos-Tafur 2006, Fig. 4 B) y en el espécimen ilustrado (ICN-MHN-CR 1867, Fig. 2 B). Esto nos permite concluir que esta cresta puede estar o no presente. En el ápice, el borde lateral puede ser recto, como en el paratipo de $H$. rotundilobata (Campos 2003, Fig. 22 E), o redondeado, como en $H$. rotundilobata (ICN-MHN-CR 1867, Fig. 2 E) e H. triangula (Ramos-Tafur 2006, Fig. 3 E); el borde cefálico del ápice puede ser liso, como en el holotipo (Rodríguez 1994, Fig. 2 C) y el paratipo (Campos 2003, Fig. 22 E) de $H$. rotundilobata, o con una hilera de pequeñas espinas, como en $H$. rotundilobata (ICN-MHNCR 1867, Fig. 2 E) e H. triangula (Ramos-Tafur 2006, Fig. 3 E).

Campos (2003) registra para $H$. rotundilobata 2 machos, cl 13.3 y 12.4 mm, cb 23.3 y $21.1 \mathrm{~mm}$ y 6 hembras, la mayor $\mathrm{cl} 13.1 \mathrm{~mm}$, cb $22.6 \mathrm{~mm}$, la menor cl $10.2 \mathrm{~mm}$, cb $17.2 \mathrm{~mm}$, de la localidad: límites entre Chocó y Valle del Cauca, Paso de Galápagos, 15 May 1985, leg. M. Alberico, CRBMUV 85147. Esta información corresponde a los datos de la etiqueta del material examinado de la colección de referencia de Biología Marina de la Universidad del Valle, CRBMUV. La determinación taxonómica de éste material se basó en la comparación del material con el paratipo, USNM 240104.

Las variaciones morfológicas descritas anteriormente para el primer gonopodo del macho corresponden a variaciones poblacionales propias de la especie, que no alteran el patrón general de $H$. rotundilobata. Así mismo, los caracteres complementarios (del caparazón, del tercer maxilípedo y de los pereopodos) son similares a los caracteres establecidos para $H$. rotundilobata. En conclusión, Hypolobocera triangula se debe considerar como sinónimo de H. rotundilobata.

Rodríguez (1994) registra como la localidad típica de Hypolobocera rotundilobata, Departamento del Chocó, Playa de Oro, alto río San Juan. Posteriormente, Campos (2003) registra la especie para límites entre Departamentos de Chocó y Valle del Cauca, Paso de Galápagos y para el Departamento del Chocó, San José del Palmar, río Ingará. El nuevo registro amplía la distribución de la especie hasta el Municipio de El Cairo en el Departamento del Valle del Cauca. Así mismo, el ámbito altitudinal inicialmente de 70 a $2000 \mathrm{~m}$, se amplia de $70 \mathrm{~m}$ a $2500 \mathrm{~m}$.

\section{AGRADECIMIENTOS}

Los autores agradecen a Celio Magalhães, del Instituto Nacional de Pesquisas da Amazonia (INPA), Brasil, por los valiosos comentarios al manuscrito.

\section{RESUMEN}

Se propone considerar las especies $H$. solimani e $H$. triangula como sinónimos de $H$. alata e $H$. rotundilobata, respectivamente. Se afirma que las diferencias morfológicas a) del primer gonopodo, del tercer maxilipedo y de 
la quela mayor entre $H$. alata e $H$. solimani y b) las diferencias del primer gonopodo entre $H$. rotundilobata e $H$. triangula, no son significativas y se originan en variaciones naturales propias de las poblaciones.

Palabras clave: Hypolobocera solimani, H. triangula, cangrejos de agua dulce, Pseudothelphusidae, Colombia, sinónimos.

\section{REFERENCIAS}

Campos, M.R. 1989. Nuevas especies de cangrejos de agua dulce del género Hypolobocera (Crustacea: Decapoda: Pseudothelphusidae) para Colombia. Trianea 3: 143-147.

Campos, M.R. 1994. Diversidad en Colombia de los cangrejos del género Neostrengeria. Academia Colombiana de Ciencias Exactas, Físicas y Naturales. Col. Jorge Alvarez Lleras. 5: 1-143.

Campos, M.R. 2003. A review of the freshwater crabs of the genus Hypolobocera Ortmann, 1897 (Crustacea: Decapoda: Brachyura: Pseudothelphusidae), from Colombia. Proc. Biol. Soc. Wash. 116: 754-802.

Campos, M.R. 2005. Freshwater crabs from Colombia. A taxonomical and distributional study. Academia Colombiana de Ciencias Exactas, Físicas y Naturales, Col. Jorge Álvares Lleras. 24: 1-363.

Campos, M.R. \& G. Rodríguez. 1993. Three new species of Strengeriana from Colombia (Crustacea: Decapoda: Pseudothelphusidae). Proc. Biol. Soc. Wash. 106: 508-513.

Ortmann, A. 1897. Carcinologische Studien. Zoologische Jahrbücher, Abteilung für Systematik, Geographie and Biologie der Tiere. 10: 258-372.
Pedraza, M. \& M.R. Campos. 2007. Estudio de la variación morfológica del gonopodo de Strengeriana maniformis (Brachyura: Pseudothelphusidae) mediante aplicación de morfometría geométrica. Caldasia. 29: 143-152.

Pretzmann, G. 1965. Vorläufiger Bericht über die Familie Pseudothelphusidae. Anz. Öster. Akad. Wiss. Math. Naturw. K1. 1: 1-10.

Ramos-Tafur, G. 2006. Dos nuevas especies de cangrejos de agua dulce (Brachyura: Pseudothelphusidae) de la Serranía de Los Paraguas, Cordillera Occidental de Colombia. Rev. Biol. Trop. 54: 1047-1056.

Rodríguez, G. 1966. The freshwater crabs of the genus Pseudothelphusa from northern Venezuela and Trinidad (Brachyura, Potamonidae). Zool. Med. Leiden. 41: 111-135.

Rodríguez, G. 1981. Decapoda. In : Hurlbert, H., G. Rodríguez \& N. D. dos Santos. Aquatic Biota of Tropical South America 1: 41-51.. San Diego State University, San Diego, California. EEUU.

Rodríguez, G. 1982. Les crabes d'eau douce d'Amérique. Famille des Pseudothelphusidae. Faune Tropicale. 22: $1-223$.

Rodríguez, G. 1994. A revision of the type material of some species of Hypolobocera and Ptychophallus (Crustacea: Decapoda: Pseudothelphusidae) in the National Museum of Natural History, Washington D. C., with descriptions of a new species and a new subspecies. Proc. Biol. Soc. Wash. 107: 296-307.

Smalley, A. 1964. A terminology for the gonopods of the American River crabs. Syst. Zool. 13: 28-31. 